\title{
Relación entre el índice de masa corporal y la frecuencia de exacerbaciones en pacientes con enfermedad pulmonar obstructiva crónica en Santa Marta, Colombia
}

\author{
Association between body mass index and exacerbation frequency in patients with chronic obstructive pulmonary \\ disease from Santa Marta, Colombia
}

John Carlos Pedrozo-Pupo' orcid.org/0000-0002-5675-7016

Gabriel Jesús Celemín-Güete' orcid.org/0000-0002-7168-0408

Adalberto Campo-Arias ${ }^{*}$ orcid.org/0000-0003-2201-7404

1 Programa de Medicina, Facultad de Ciencias de la Salud, Universidad del Magdalena. Santa Marta, Colombia

Fecha de recepción: Abril 8 - 2018

Fecha de revisión: Febrero 12 - 2019

Fecha de aceptación: Abril 22 - 2019

Pedrozo-Pupo JC, Celemín-Güete GJ, Campo-Arias A. Relación entre el índice de masa corporal y la frecuencia de exacerbaciones en pacientes con enfermedad pulmonar obstructiva crónica en Santa Marta, Colombia. Univ. Salud. 2019;21(2):127-131. DOI: http://dx.doi.org/10.22267/rus.192102.146

\section{Resumen}

Introducción: El índice de masa corporal (IMC) se asocia inversamente a la frecuencia de exacerbaciones en pacientes con enfermedad pulmonar obstructiva crónica (EPOC); sin embargo, esta puede variar según el contexto. Objetivo: Cuantificar la asociación entre el IMC y la frecuencia de exacerbaciones en pacientes en Santa Marta, Colombia. Materiales y métodos: Estudio transversal de adultos con EPOC. Se calculó el IMC y la frecuencia de exacerbaciones se estimó a partir de la clasificación GOLD de estado global. Resultados: Participaron 292 pacientes entre 49 y 95 años; 61,6\% eran hombres. Los IMC se observaron entre 12,8 y 40,2 (media=24,2; $D E=4,5)$ distribuidos en 21 pacientes $(7,2 \%)$ con desnutrición; 153 (52,4\%), saludables; y $118(40,4 \%)$, sobrepeso-obesidad. Un total de 146 pacientes (53,4\%) se clasificaron GOLD A o B (exacerbaciones no frecuentes); y 136 pacientes (46,6\%), GOLD C o D (exacerbaciones frecuentes). El 85,7\% de los pacientes con desnutrición presentaron exacerbaciones frecuentes comparado con 51,6\% en pacientes con peso saludable y 33,1\% en pacientes con sobrepeso-obesidad (OR=0,18; IC95\% 0,05-0,66 para peso saludable y OR=0,08; IC95\% 0,02-0,29 para sobrepeso-obesidad frente a desnutrición). Conclusiones: El IMC presenta una relación inversa con la frecuencia de exacerbaciones en pacientes con EPOC de Santa Marta, Colombia.

Palabras clave: Enfermedad pulmonar obstructiva crónica; índice de masa corporal; calidad de vida; estudios transversales. (Fuente: DeCS, Bireme).

\begin{abstract}
Introduction: Body mass index (BMI) is inversely related to the exacerbation frequency in patients with chronic obstructive pulmonary disease (COPD). However, this relationship may vary depending on the context. Objective: To quantify the association of BMI with exacerbation frequency in patients from Santa Marta-Colombia. Materials and methods: A crosssectional study of adults with COPD. We calculated the BMIs, and the exacerbation frequencies were estimated using the Global initiative for Chronic Obstructive Lung Disease (GOLD) classification. Results: 292 patients aged between 49-95 years were included. From those, 180 (61.6\%) were male, 153 (52.4\%) were healthy, 21 (7.2\%) showed malnutrition, and 118 $(40.4 \%)$ were overweight/obese patients. The observed IMCs were between 12.8 and 40.2 (median=24.2; $S D=4.5$ ). Whereas 156 patients $(53,4 \%)$ were classified as GOLD A or B (infrequent exacerbations), 136 of them (46.6\%) were GOLD C of D (frequent exacerbations). 85,7\% of the patients with malnutrition showed frequent exacerbations, compared to both patients with healthy weight $(51,6 \%)$ and overweight/obese patients $(33.1 \%)(\mathrm{OR}=0.18$; CI95\% 0.05-0.66 for healthy weight and $\mathrm{OR}=0.08$; CI95\% 0.02-0.29 for overweight/obesity, both compared to patients with malnutrition). Conclusions: BMI is inversely correlated with exacerbation frequency in patients with COPD from Santa Marta-Colombia.
\end{abstract}

Keywords: Chronic pulmonary obstructive disease; body mass index; quality of life; cross-sectional studies. (Source: DeCS, Bireme). 


\section{Introducción}

La prevalencia de la enfermedad pulmonar obstructiva crónica (EPOC) es variable, por ejemplo en Ciudad de México es del 7\%, en Montevideo del 19\%; $y$ en Colombia el estudio PREPOCOL (Prevalencia de EPOC en Colombia) informó una prevalencia del 8,9\%(1). Según la iniciativa GOLD (Global Initiative for Chronic Obstructive Lung Disease), la EPOC es una enfermedad común, prevenible y tratable que se caracteriza por síntomas respiratorios persistentes y limitación del flujo aéreo debido a anomalías de las vías respiratorias y/o alveolares; generalmente causadas por una exposición significativa a partículas o gases nocivos(2) No obstante la prevalencia de la EPOC, con frecuencia el diagnóstico se realiza en etapas tardías(3).

El estado nutricional o el índice de masa corporal (IMC) afectan el curso y el pronóstico, la calidad de vida y la mortalidad, de los pacientes con EPOC(4-6). En este grupo de pacientes, como en otros pacientes con enfermedades cardiovasculares, el IMC está inversamente relacionado con la calidad de vida(4,7,8).

La gradación GOLD realiza una valoración integral del paciente con EPOC, considera la limitación al flujo de aire, el impacto sobre el estado de salud del paciente (síntomas respiratorios), y el riesgo de eventos a futuro, como exacerbaciones, clasifica al paciente en cuatro categorías, A, B, C y D, menor a mayor gravedad(2).

Los estudios precedentes mostraron una asociación consistente entre la obesidad y las gradaciones I a IV de GOLD (valor aislado del VEF1) con las comparaciones con estadística paramétrica ${ }^{(9-14)}$. Sin embargo, los hallazgos de la asociación son divergentes o difíciles de interpretar en aquellos análisis realizados con una aproximación no paramétrica(15-18).

En consecuencia, dada la reciente introducción de la nueva clasificación GOLD (evaluación combinada) se cuenta con un único estudio que muestra datos empíricos de la asociación de la frecuencia de exacerbaciones basada en esta clasificación y el IMC. Lambert, et al., compararon en el peso (normal o sobrepeso y las tres clases de obesidad) y observaron asociaciones que no siguieron un patrón ordinal. Los pacientes con peso normal o sobrepeso mostraron más en la evaluación GOLD en la A y $\mathrm{C}$ que los pacientes con cualquier clase de obesidad. Sin embargo, los pacientes con obesidad fueron con mayor frecuencia calificados en la evaluación B de GOLD que los pacientes con peso normal o sobrepeso(15).

Sin duda, el conocimiento de la asociación entre el IMC y la nueva reagrupación de GOLD permitirá considerar el estado nutricional no sólo como un indicador de pronóstico general de la EPOC sino también en el proceso de rehabilitación para mejorar la funcionalidad y la calidad de vida de los pacientes(19-20).

El objetivo de esta investigación fue cuantificar la asociación entre el IMC y la frecuencia de exacerbaciones en pacientes ambulatorios con EPOC en Santa Marta, Colombia.

\section{Materiales y métodos}

\section{Diseño, población y muestra}

Se diseñó un estudio cuantitativo, analítico, transversal. Se solicitó la participación de pacientes con EPOC atendidos en diferentes instituciones de Santa Marta. Se tomó una muestra no probabilística, por conveniencia, por la secuencia de atención, desde enero hasta mediados de diciembre de 2016. No obstante, se espera contar por lo menos con 267 pacientes, número de pacientes requeridos para una prevalencia esperada del $25 \%$ de exacerbaciones frecuentes (GOLD C y D), margen de error de 5 y nivel de confianza del $95 \%{ }^{(22)}$. De la misma forma, este número de participantes permitía realizar el ajuste de este número de participantes hasta seis variables en bloque, bajo el principio de que contaron por lo menos 67 casos con exacerbaciones frecuentes, a la razón de 10 casos por cada variable de confusión(23). Se incluyó pacientes mayores de edad atendidos en instituciones con servicio de consulta externa de neumología, independientemente de otra característica. Se excluyeron los pacientes que no completaron toda la evaluación.

\section{Mediciones}

Se tomaron los datos demográficos básicos además de peso, talla y frecuencia de exacerbaciones basadas en la clasificación de GOLD. El índice de masa corporal se calculó según la fórmula de Quetelet a partir del peso y altura (Fórmula 1).

$$
\mathrm{IMC}=\text { peso }(\mathrm{kg}) / \text { talla }\left[\mathrm{m}^{2}\right]^{(24)}
$$


Según el IMC los pacientes se categorizaron en cuatro grupos: Desnutrición: $18,4 \mathrm{~kg} / \mathrm{m}^{2}$ o menos; saludable entre 18,5 y $24,9 \mathrm{~kg} / \mathrm{m}^{2}$; sobrepeso entre 25,0 y 29,9 $\mathrm{kg} / \mathrm{m}^{2} ;$ y obesidad: $30,0 \mathrm{~kg} / \mathrm{m}^{2}$ o más.

Para precisar el diagnóstico de EPOC se consideró la presencia los síntomas clave como disnea, tos y producción de esputo en paciente con una función pulmonar sugestiva de la condición. Asimismo, se revisaron antecedentes de exposición al cigarrillo o humo de leña, en consonancia con la guía GOLD(2). Las espirometrías se llevaron a cabo con el uso de un espirómetro (Master Screen PFT System de CareFusion $^{\circledR}$ - EU). La medición se realizó con los criterios y procedimientos recomendados por American Thoracic Society/European Respiratory Society (ATS/ERS) para la estandarización de la evaluación(25).

La clasificación GOLD incluye los hallazgos en espirometría, la evaluación con el COPD Assessment Test (CAT) (EPOC por sus sigla en inglés) que aborda gravedad de síntomas y desempeño en actividades cotidianas y sociales y exacerbaciones(2). Para analizar estas gradaciones se definieron dos grupos; menor frecuencia de exacerbaciones para las categorías A y B; y mayor frecuencia de exacerbaciones, $\mathrm{C}$ y D.

Los pacientes diagnosticados con EPOC, atendidos en los diferentes centros médicos fueron identificados e invitados a participar en el estudio. Posteriormente, los pacientes fueron evaluados todos en una de las instituciones por uno de los investigadores (JCPP). La evaluación incluyó completar los componentes de la iniciativa GOLD.

\section{Análisis estadístico}

Para el análisis descriptivo se frecuencias y porcentajes para los datos nominales y para datos cuantitativos, media y desviación estándar (DE). Se calcularon las razones de momios (OR), con los respectivos intervalos de confianza de 95\% (IC 95\%) para datos ordenados mediante regresión logística multinomial. Se tomó como independiente el IMC y como variable dependiente la frecuencia de exacerbaciones, menor y mayor número. Las asociaciones establecieron con el paquete IBM-SPSS Statistics versión 22.

\section{Consideraciones éticas}

Se contó la revisión y aprobación del comité de ética de la Facultad de Ciencias de la Salud de la Universidad del Magdalena. Los participantes firmaron consentimiento informado una vez conocieron los objetivos del estudio y que la participación no representaba un riesgo mayor para la integridad física y emocional que la revisión clínica rutinaria para la EPOC, todo en consonancia con la Resolución 8430 de 1993 del Ministerio de Salud(21).

\section{Resultados}

Participaron 292 pacientes en edades entre 49 y 95 años (media=73,5; DE=8,7); 180 hombres $(61,6 \%$ ) y 112 mujeres $(38,4 \%)$. Los IMC se observaron entre 12,8 y 40,2; media 24,2 (DE=4,5); en forma ordinal los pacientes se clasificaron de la siguiente manera: 21 $(7,2 \%)$ con desnutrición; $153(52,4 \%)$ con peso saludable; y $118(40,4 \%)$ con sobrepeso u obesidad. Los pacientes con sobrepeso y obesidad se incluyeron en la misma categoría porque no se observó diferencia estadísticamente significativa entre los dos grupos en relación con la categoría GOLD, $\mathrm{OR}=1,4$; IC95\% 0,6-3,8.

Se contó mayor número de exacerbaciones (GOLD C y D) en 136 pacientes $(46,6 \%)$ y menor frecuencia de exacerbaciones (GOLD A y B) en 156 (53,4\%). El $85,7 \%$ de los pacientes con desnutrición presentaron mayor frecuencia de exacerbaciones comparado con el $51,6 \%$ en pacientes con peso saludable y $33,1 \%$ en pacientes con sobrepeso u obesidad. La diferencia fue estadísticamente significativa (Chi cuadrado $=23,2$; $\mathrm{gl}=2 ; \mathrm{p}=0,001$ y regresión multinomial, $\mathrm{OR}=0,18$; IC95\% 0,05-0,66 para peso saludable y $\mathrm{OR}=0,08$; IC95\% 0,02-0,29 para sobrepeso u obesidad frente a desnutrición).

\section{Discusión}

En la presente investigación se observa que el IMC o estado nutricional muestra una asociación inversa con la frecuencia de exacerbaciones en pacientes ambulatorios con EPOC en Santa Marta, Colombia. Este hallazgo es parcialmente congruente con lo observado en el estudio de Eriksson, et al., quienes mostraron que los pacientes con desnutrición fueron calificados GOLD 3-4, en la anterior versión de la gradación, que los pacientes con peso normal(16). Sin embargo, esta observación es contraria a lo reportado por Lambert, et al., quienes mostraron que en 
conjunto los pacientes con mayor IMC (obesos) presentaron con mayor frecuencia clase GOLD D que los pacientes con peso normal o sobrepeso(15). Por otro lado, McCormack, et al., observaron en una muestra de 84 pacientes que el IMC (peso normal y obesidad) no se asoció a la categoría GOLD(17); esta independencia la replicaron también Koniski, et al., con una muestra de 996 pacientes, donde encontraron que el IMC no se relacionó significativamente con la clase GOLD(18).

La inconsistencia en la asociación entre IMC y clasificación GOLD con el uso de estadística no paramétrica se puede explicar en gran parte por las características demográficas y clínicas de la población(26). Asimismo, se debe considerar la línea de causalidad y tener presente que la asociación no necesariamente es causación(27). Es posible que la pérdida de peso en pacientes con EPOC gradados en GOLD C y D sea en sí mismo un indicador de deterioro funcional y no la causa de éste, es decir, no es la falta de peso por pérdida de masa muscular o grasa corporal responsable del deterioro de la función pulmonar, el funcionamiento global y la mortalidad del paciente con $\operatorname{EPOC}(6,19,28)$. De igual manera, considerar la naturaleza arbitraria de la clasificación GOLD que ha mostrado problemas por no seguir una secuencia lineal de menor a mayor frecuencia de exacerbaciones, $\quad \mathrm{A}<\mathrm{B}<\mathrm{C}<\mathrm{D} \quad \mathrm{y}$ las variaciones importantes que se dan en la clasificación según la escala usada para cuantificar los síntomas $(29,30)$.

Este trabajo tiene la ventaja de precisar la asociación entre el IMC y la frecuencia de exacerbaciones en pacientes con EPOC. No obstante, tiene la limitación de un tamaño de muestra reducido para ajustar la asociación entre IMC y la frecuencia de exacerbaciones por posibles variables de confusión como la edad(26). Futuras investigaciones deben considerar este punto.

\section{Conclusiones}

El IMC o el estado nutricional presentan una relación inversa con la frecuencia de exacerbaciones en pacientes con EPOC de Santa Marta, Colombia. Se recomienda realizar más investigaciones con muestras mayores que corroboren esta observación.

\section{Conflicto de intereses}

Ninguno declarado por los autores.

\section{Referencias}

1. Caballero A, Torres-Duque CA, Jaramillo C, Bolívar F, Sanabria F, Osorio $\mathrm{P}$, et al. Prevalence of COPD in five Colombian cities situated at low, medium, and high altitude (PREPOCOL study). Chest. 2008;133(2):343-9. doi 10.1378/chest.07-1361

2. Global Initiative for Chronic Obstructive Lung Disease. Global Initiative for Chronic Obstructive, 2017. https://goldcopd.org/ (acceso: 08-03-2018).

3. Moreira GL, Manzano BM, Gazzotti MR, Nascimento OA, Perez-Padilla R, Menezes AM, et al. PLATINO, a nine-year follow-up study of COPD in the city of São Paulo, Brazil: the problem of underdiagnosis. J Bras Pneumol. 2014;40(1):307. doi 10.1590/S1806-37132014000100005

4. Schols A, Broekhuizen R, Weling-scheepers CA, Wouters EF. Body composition and mortality in chronic obstructive pulmonary disease. Am J Clin Nutr. 2005;81(1):53-9. doi: 10.1093/ajcn.82.1.53

5. Poulain M, Doucet M, Major GC, Drapeau F, Sériès F, Boulet LP, et al. The effect of obesity on chronic respiratory diseases: Pathophysiology and therapeutic strategies. Can Med Assoc J. 2006;174(9):1293-9. doi: 10.1503/cmaj.051299

6. Cao C, Wang R, Wang J, Bunjhoo $\mathrm{H}, \mathrm{Xu} \mathrm{Y}$, Xiong W. Body mass index and mortality in chronic obstructive pulmonary disease: A meta-analysis. PLoS One. 2012;7(8):e43892. doi: 10.1371/journal.pone.0043892

7. Fernández A, Ferrante D, Hrabar A, Soifer S, Varini S, Nul D. Valor pronóstico del índice de masa corporal en pacientes con insuficiencia cardíaca crónica : Registro GESICA. Rev Am Med Respir. 2006;74(4):204-10.

8. Niedziela J, Hudzik B, Niedziela N, Gasior M, Gierlotka M Wasilewski J, et al. The obesity paradox in acute coronary syndrome: a meta-analysis. Eur J Epidemiol. 2014;29(11):801-12. doi: 10.1007/s10654-014-9961-9

9. Montes de Oca M, Tálamo C, Perez-Padilla R, Jardim JR, Muiño A, Lopez MV, et al. Chronic obstructive pulmonary disease and body mass index in five Latin America cities: The PLATINO study. Respir Med. 2008;102(5):642-50. doi: 10. .1016/j.rmed.2007.12.025

10. De S. Body mass index among patient with chronic obstructive pulmonary diseases. Indian J Physiol Pharmacol. 2012;56(4):353-8.

11. Mitra M, Ghosh S, Kaushik S, Saha A, Panchadhyayee $P$, Biswas A, et al. A study of correlation between body mass index and GOLD staging of chronic obstructive pulmonary disease patients. J Assoc Chest Physicians. 2013;1(2):58-61. doi: $10.4103 / 2320-8775.123217$

12. Garciá-Rio F, Soriano JB, Miravitlles M, Munõz L, DuranTauleria E, Sańchez G, et al. Impact of obesity on the clinical profile of a population-based sample with chronic obstructive pulmonary disease. PLoS One. 2014;9(8):1-10. doi: 10.1371/journal.pone.0105220

13. Feroz A, Dawar S, Swami S, Bhangoo HS, Soothwal P. Can body mass index correlate with severity of chronic obstructive pulmonary disease? A cross sectional study done in rural population of Jaipur. Indian J Basic Appl Med Res. 2015;5(3):696-703.

14. Assal HH, Kamal E. Body mass index and its relation to GOLD stage in chronic obstructive pulmonary disease patients. Egypt J Chest Dis Tuberc. 2016;65(2):411-4. doi: 10.1016/j.ejcdt.2016.01.002 
15. Lambert AA, Putcha N, Drummond MB, Boriek AM, Hanania NA, Kim V, et al. Obesity is associated with increased morbidity in moderate to severe COPD. Chest. 2017;151(1):68-77. doi: 10.1016/j.chest.2016.08.1432

16. Ericksson B, Backman $\mathrm{H}$, Bossios A, Bjerg A, Hedman L, Lindberg A, et al. Only severe COPD is associated with being underweight: results from a population survey. ERJ Open Res. 2016;2(3):00051-2015. doi: 10.1183/23120541.000512015.

17. McCormack MC, Belli AJ, Kaji DA, Matsui EC, Brigham EP, Peng RD, et al. Obesity as a susceptibility factor to indoor particulate matter health effects in COPD. Eur Respir J. 2015;45(5):1248-57. doi: 10.1183/09031936.00081414

18. Koniski ML, Salhi H, Lahlou A, Rashid N, El Hasnaoui A. Distribution of body mass index among subjects with COPD in the Middle East and North Africa region: Data from the BREATHE study. Int J COPD. 2015;10(1):1685-94. doi: 10.2147/COPD.S87259

19. Alvis SA, Gómez MV, Flórez JS. Caracterización de la capacidad funcional y calidad de vida de los pacientes con EPOC en un centro de rehabilitación pulmonar integral de Cartagena. Cienc Salud. 2014;3(10):17-24. doi: https://doi.org/10.21774/cys.v3i10.455

20. Hanson C, Rutten EP, Wouters EF, Rennard S. Influence of diet and obesity on COPD development and outcomes Introduction to the prevalence of obesity. Int J Chron Obstruct Pulmon Dis. 2014;9:723-33. doi: 10.2147/COPD.S50111
21. Ministerio de Salud de Colombia. Resolución 8430 de 1993. Bogotá: MinSalud; 1993.

22. Hernández J. Selección del tamaño de muestra en estudios clínicos. Rev Colomb Gastroenterol. 2006;21(6):118-21.

23. Katz MH. Study Design and Statistical Analysis: Univariate statistics. First. En: Katz MH, editor. Cambridge: Cambridge University Press; 2004. p. 91-112.

24. de Souza W, Tajes D, Gomes L, Grzelczak M. Índice de adiposidade corporal (IAC) como predictor de gordura corporal: um estudo de revisáo. Saude Meio Ambient. 2015;4(1):32-8. doi: 10.24302/sma.v4i1.638

25. Rush L. Improving interpretation of spirometry for better diagnosis. Prim Heal Care. 2018;28(2):34-41. doi: 10.7748/phc.2018.e1367

26. Sedgwick P. Bias in observational study designs: Cross sectional studies. BMJ. 2015;350:h1286. doi: 10.1136/bmj.h1286

27. Kaplan D. Causal inference for observational studies. J Infect Dis. 2019;219(1):1-2. doi: 10.1093/infdis/jiy392

28. Goto T, Tsugawa Y, Faridi MK, Camargo CA Jr, Hasegawa K. Reduced risk of acute exacerbation of COPD after bariatric surgery. Chest. 2017;153(3):611-7. doi: 10.1016/j.chest.2017.07.003

29. Einarson TR, Bereza BG, Nielsen TA, Hemels ME. Utilities for asthma and COPD according to category of severity: a comprehensive literature review. J Med Econ. 2015;18(7):550-63. doi: 10.3111/13696998.2015.1025793

30. Sívori M, Fernández R. Clasificación de EPOC GOLD 2018. Rev Am Med Respir. 2018;18(2):140-2. 